\title{
Uveitis and Multiple Sclerosis: Potential Common Causal Mutations
}

\author{
Alejandra de-la-Torre ${ }^{1}$ (D) Claudia T. Silva-Aldana ${ }^{2} \cdot$ Juliana Muñoz-Ortiz $^{1,3} \cdot$ Laura B. Piñeros-Hernández $^{2}$. \\ Oscar Otero ${ }^{3}$ - Alejandra Domínguez ${ }^{1}$. León A. Faciolince ${ }^{1,3}$. Mauricio Arcos-Holzinger ${ }^{1}$. Claudio Mastronardi ${ }^{4}$. \\ Nora Constanza Contreras-Bravo ${ }^{2} \cdot$ Carlos Martín Restrepo $^{2} \cdot$ Mauricio Arcos-Burgos $^{5}$
}

Received: 11 March 2019 / Accepted: 26 April 2019 / Published online: 3 June 2019

(C) The Author(s) 2019

\begin{abstract}
Uveitis, defined as inflammation of the uveal tract of the eye, is a leading cause of blindness and visual impairment throughout the world. The etiology of uveitis is complex, and autoimmunity plays a major role in its pathogenesis. Intermediate uveitis (IU), a subtype of ocular inflammation, has been associated with systemic autoimmune disorders, specifically with multiple sclerosis (MS). This article reports a rare three-generation family with several members affected by IU (four siblings) and comorbid MS (two siblings fulfilling MS diagnostic criteria and a third sibling presenting some neurological symptoms). Based on the clinical findings, we captured and sequenced whole exomes of seven pedigree members (affected and unaffected). Using a recessive model of transmission with full penetrance, we applied genetic linkage analysis to define minimal critical regions (MCRs) in suggestive or nominal regions of linkage. In these MCRs, we defined functional (some pathogenic), novel, and rare mutations that segregated as homozygous in affected and heterozygous in unaffected family members. The genes harboring these mutations, including $D G K I$, TNFRSF10A, GNGT1, CPAMD8, and BAFF, which are expressed in both eye and brain tissues and/or are related to autoimmune diseases, provide new avenues to evaluate the inherited causes of these devastating autoimmune conditions.
\end{abstract}

Keywords Uveitis $\cdot$ Multiple sclerosis $\cdot$ Genetics $\cdot$ Mutations $\cdot$ Whole exome sequencing $\cdot$ Pedigree

\section{Introduction}

Uveitis is inflammation of the eye's uveal tract, which includes the iris, ciliary body, and choroid [1]. The annual

Alejandra de-la-Torre, Claudia T. Silva-Aldana and Mauricio ArcosBurgos contributed equally to this work.

Alejandra de-la-Torre

alejadelatorre@yahoo.com

Mauricio Arcos-Burgos

mauricio.arcos@udea.edu.co

1 Grupo de investigación en neurociencias (NEUROS), Escuela de Medicina y Ciencias de la Salud, Universidad del Rosario, Bogotá, Colombia

2 Center For Research in Genetics and Genomics-CIGGUR, GENIUROS Research Group, School of Medicine and Health Sciences, Universidad del Rosario, Bogotá, Colombia

3 Escuela Superior de Oftalmología, Instituto Barraquer de América, Bogotá, Colombia

4 INPAC Research Group, Fundación Universitaria Sanitas, Bogotá, Colombia

5 Grupo de Investigación en Psiquiatría (GIPSI), Departamento de Psiquiatría, Instituto de Investigaciones Medicas (IIM), Facultad de Medicina, Universidad de Antioquia, Medellin, Colombia incidence of uveitis varies between 17.4 and 52.4 new cases per 100,000 , and the prevalence is between 38 and 714 per 100,000 . This disease causes $2.8-10 \%$ of all cases of blindness and visual impairment worldwide, and severity depends on factors such as chronicity and whether or not adjacent tissues, such as the retina, optic nerve, and vitreous, are affected [2-5].

The Uveitis Nomenclature Standardization (SUN) Working Group and the International Uveitis Study Group (IUSG) classify uveitis according to the anatomical location of the inflammatory process, i.e., anterior uveitis (iritis, iridocyclitis, and anterior cyclitis), intermediate uveitis (pars planitis, posterior cyclitis, and hyalitis), posterior uveitis (focal, multifocal, or diffuse choroiditis, chorioretinitis, retinitis, and neuroretinitis), and panuveitis (anterior chamber, vitreous, retina, and choroid) [6-9].

The etiology of uveitis is complex and involves confined autoimmune processes [10], systemic autoimmune diseases (e.g., multiple sclerosis, Behçet's disease), infectious diseases (e.g., Toxoplasma gondii, Mycobacterium tuberculosis, Herpes virus, and Treponema pallidum), and inherited genetic susceptibility [11-13]. Previous studies reported association of the susceptibility to develop uveitis with polymorphisms in the human lymphocyte 
antigen (HLA) class II genes, interleukins 10 and 6 (IL10 and $I L 6$, respectively), tumoral necrosis factor $(T N F)$, transforming growth factor beta 1 and 2 (TGFB1 and $T G F B 2$, respectively), transforming growth factor beta receptor 3 (TGFBR3), interferon gamma (INFG), interleukin 2 receptor subunit alpha $(I L 2 R A)$, and cytotoxic $\mathrm{T}$ lymphocyte protein 4 (CTLA4), among others [11].

In this article, specific focus is brought to intermediate uveitis (IU) which is the most frequent type of uveitis associated with MS (61-80\%) [14-16]. One explanation attributes this association to the ontogenetic relationship between nervous and ocular tissues. An example of this is the MS demyelination process, which affects both the central nervous system and neuroophthalmic tracts [16] producing histopathological and clinical findings common to IU and MS. These include $\mathrm{T}$ cells, especially $\mathrm{T}$ helper lymphocytes (Th), which infiltrate areas surrounding retinal vessels creating pathognomonic histopathological changes known as "snow banking" or "strings of pearls" [17]. Interestingly, patients with MS have autoreactive $\mathrm{T}$ cells and antibodies directed against glial proteins that are also detected in snow banking formations. Some authors explain this by the presence of autoreactive $\mathrm{T}$ cells directed toward a common glial epitope present in MS and/or IU patients [18].

Given that IU and MS are rare disorders, it is unusual to find patients suffering from both conditions and even less common to find several siblings affected by both conditions. In this manuscript, we report a pedigree with four siblings affected by IU of which two present sufficient symptoms for the diagnosis of MS (as stated by the revised 2017 McDonald criteria for the diagnosis of MS) and one presents neurological symptoms that do not fulfill the McDonald criteria. We hypothesize that rare/novel genetic variants of major effect shape shared genetic susceptibility to both IU and MS. To test this hypothesis, we applied whole exome capture and sequencing and used the resulting genomic variation to determine genetic linkage of potential novel and rare pathogenic causal mutations with the phenotype.

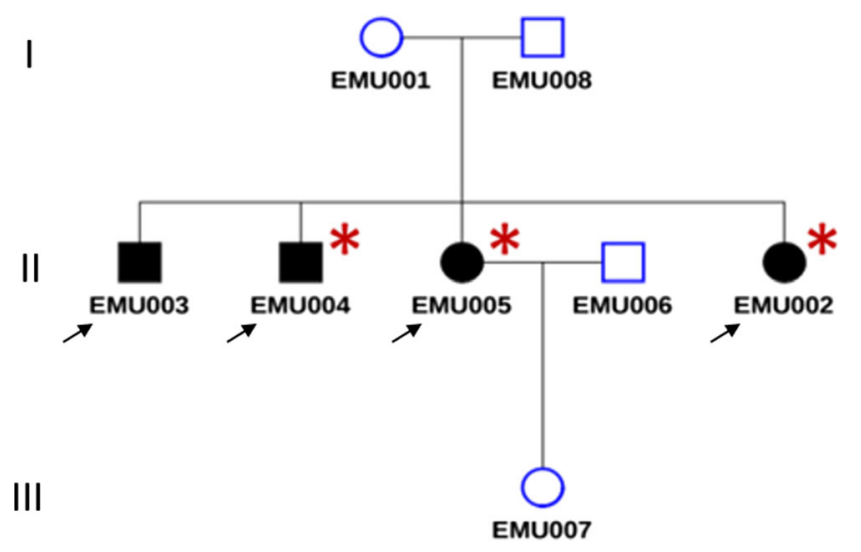

Fig. 1 Genealogy segregating intermediate uveitis (IU) and multiple sclerosis (MS). Intermediate uveitis (black signals condition of interest). Comorbid multiple sclerosis (asterisk)

\section{Methods}

\section{Patients}

We studied three generations of a family composed of eight individuals. Four members of the family were affected by IU (EMU003, EMU004, EMU005, and EMU002) of which two also fulfilled diagnostic criteria of MS (EMU005 and EMU002), and a third had neurological symptoms suggesting MS comorbidity (EMU004) (Fig. 1). Briefly, the family is composed of unaffected parents, four siblings, two women and two men, the husband of one of the women, and a granddaughter. IU in all siblings began in childhood (6 to 12 years old) with episodes of ocular inflammation. A more aggressive development of the disease, with a higher incidence of complications, including retinal detachment, was observed in women (Fig. 2). Consequently, more intensive therapies were applied to females (higher doses of steroids and immunosuppressive drugs, such as methotrexate) (Table 1). Three of the siblings developed neurologic symptoms, including paresthesia and muscular weakness (Table 2). Magnetic resonance imaging of the brain and cervical spine showed the presence of periventricular-demyelinating plaques in affected women (Figs. 3, 4, and 5). Similar to the ocular disease, the neurological symptoms were more aggressive in women than in men

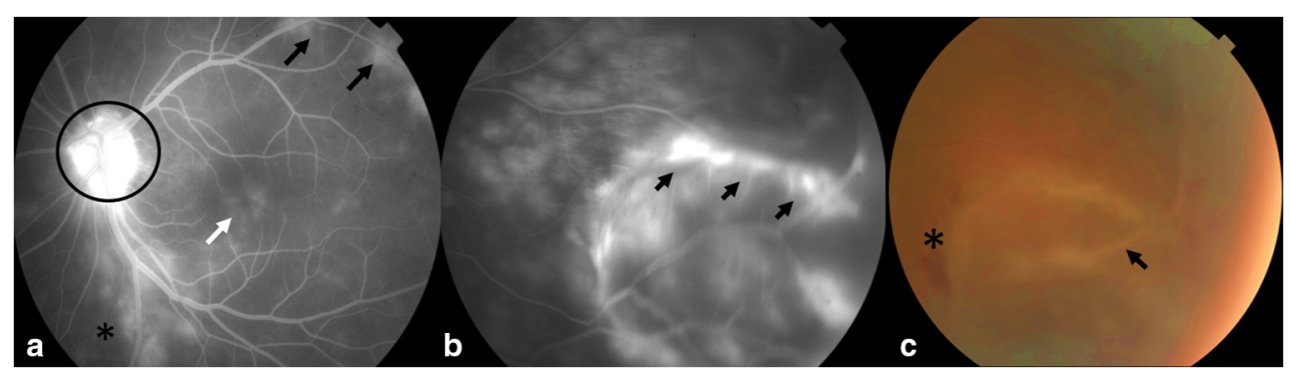

Fig. 2 Retinal fluorescein angiography and fundus photography EMU005. a Papillitis (circle), cystoid macular edema (white arrow), perivasculitis (black arrows), and retinitis (asterisk). b Exudative retinal detachment (black arrows). c Intraretinal hemorrhage (asterisk) and retinal detachment (black arrow) 
(multiple hospitalizations and treatment with interferon were necessary for women). Females EMU005 and EMU002 fulfilled the revised $2017 \mathrm{McD}$ onald criteria for the diagnosis of MS (Table 2).

\section{Whole Exome Capture, Sequencing, and Bioinformatic Analysis}

Three methods were used to quantify and qualify DNA: (1) DNA purity was checked using a NanoDrop spectrophotometer (Thermo Scientific, Waltham, MA, USA) (OD 260/280 ratio); (2) DNA degradation and contamination were monitored on $1 \%$ agarose gels; (3) DNA concentration was measured using a Qubit fluorometer (Thermo Scientific, Waltham, MA, USA).

DNA samples with OD 260/280 ratios between 1.8 and 2.0 and concentration above $1.0 \mu \mathrm{g}$ were used to prepare sequencing libraries. Library preparation for sequencing: Liquid-phase hybridization using Agilent SureSelect Human All ExonV5/V6 (Agilent Technologies, Santa Clara, CA, USA) was applied according to the manufacturer's instructions to efficiently enrich whole exons, which were sequenced on an Illumina platform. Next-generation sequencing: Genomic DNA was randomly fragmented to 180-280 bp with Covaris cracker (Covaris, Woburn, MA, USA), and then, DNA fragments were end polished, A-tailed, and ligated with the full-length adapter for Illumina sequencing. Fragments with specific indexes were hybridized with more than 543,872 biotin-labeled probes after pooling; then, magnetic beads with streptomycin were used to capture 334,378 exons from 20,965 genes. After PCR amplification and quality control, libraries were sequenced. Bioinformatic analysis: All sequenced data were quality assessed (base quality distribution, nucleotide distribution, and presence of adapters, chimeras, and other contaminants) to identify and remove low-quality data and samples from further analysis. All high-quality data was then mapped to the human genome assembly using the bwa-mem algorithm [19]. Aligned files were processed using Genome Analysis Tool Kit (GATK) [20] for base quality recalibration, insertion-deletion (indel) realignments, and duplicate removal. This was followed by single nucleotide polymorphism (SNP) and indel discovery and genotyping (plus phasing where applicable) according to GATK Best Practices recommendations [21, 22]. All variant calls were subject to variant quality score recalibration and filtering to remove low-quality variants. Remaining highquality variants were annotated for predicted functional consequences using the Voting Report Index, which includes SIFT, PolyPhen2 HVAR, Mutation Taster, Mutation Assessor, FATHMM, and FATHMM MKL Coding. For a conservative filter, variants were kept that had none, one, or maybe two tolerated predictions. A more conservative filter would keep variants based on three, four, or five damaging 


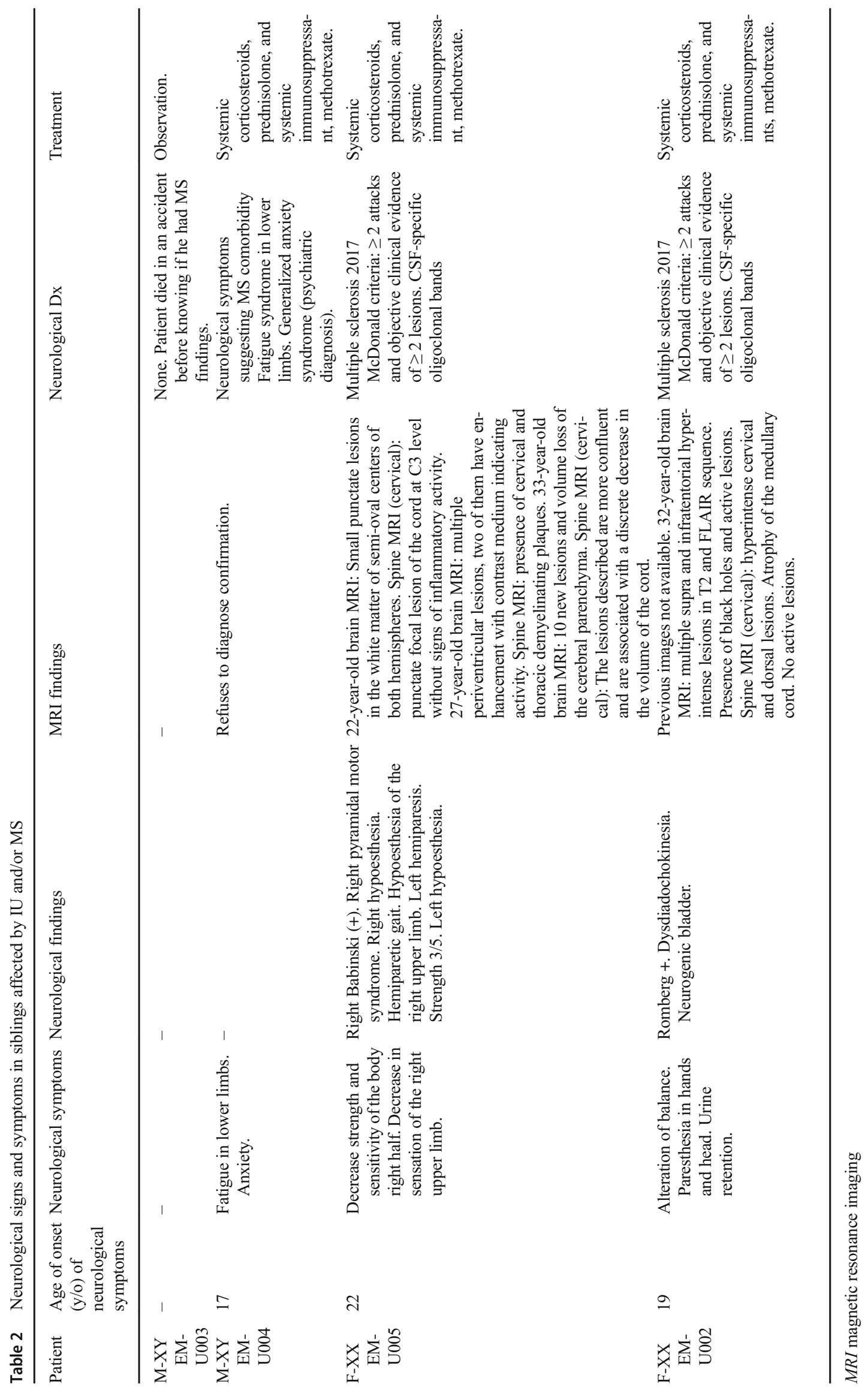


Fig. 3 Brain IRM FLAIR sequence year 2007 (a), 2011 (b), and 2012 (c). Bilateral progressive periventricular hyperintensities around the posterior horns of the lateral ventricles (white arrows)

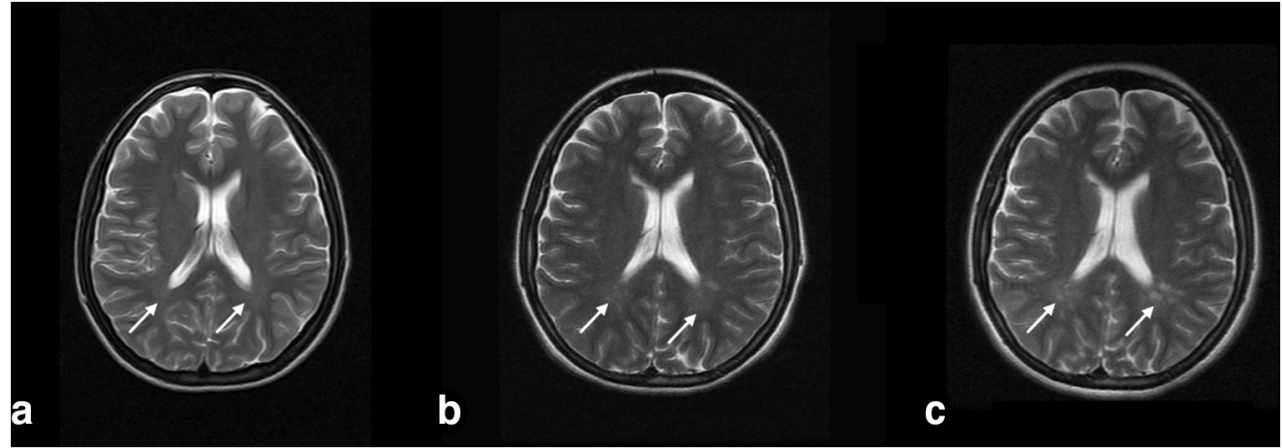

predictions. Many variants do not have five algorithms with non-missing values. Updated annotations from the NCBI 1000 genome project were used to evaluate novelty and rareness of variants.

Linkage Analysis Linkage analysis to determine cosegregation of genomic regions with phenotype was performed using Superlink (http://cbl-hap.cs.technion.ac.il/superlink-snp/ main.php). Loci of interest were suggested by single-marker and multipoint linkage using parametric and non-parametric analyses with polymorphic SNPs genotyped by whole exome sequencing. Markers were combined in subsets of two, three, and four, with the trait locus moving across the marker map. Marker positions were adapted from the position of the SNP according to HGM37. The trait allele frequency was set at 0 . 01. Averaging in 50:50 proportions set the marker allele frequencies. As recommended by other authors, the use of a 50: 50 mixture is a good and cautious choice that avoids inflating LOD scores for alleles that are rare in controls [23]. As inheritance and penetrance models, we used the segregation analysis implemented in Superlink. LOD scores were maximized for alleles with higher likelihood.

Fig. 4 a Contrasted brain MRI T1 sequences (2017). High uptake images along the supramarginal gyrus in the caudal aspect of the left parietal lobe (white arrows). b No contrasted brain MRI T1 sequences (2017). Hypointense sphere-like image in the caudal aspect of the left parietal lobe (white arrows)

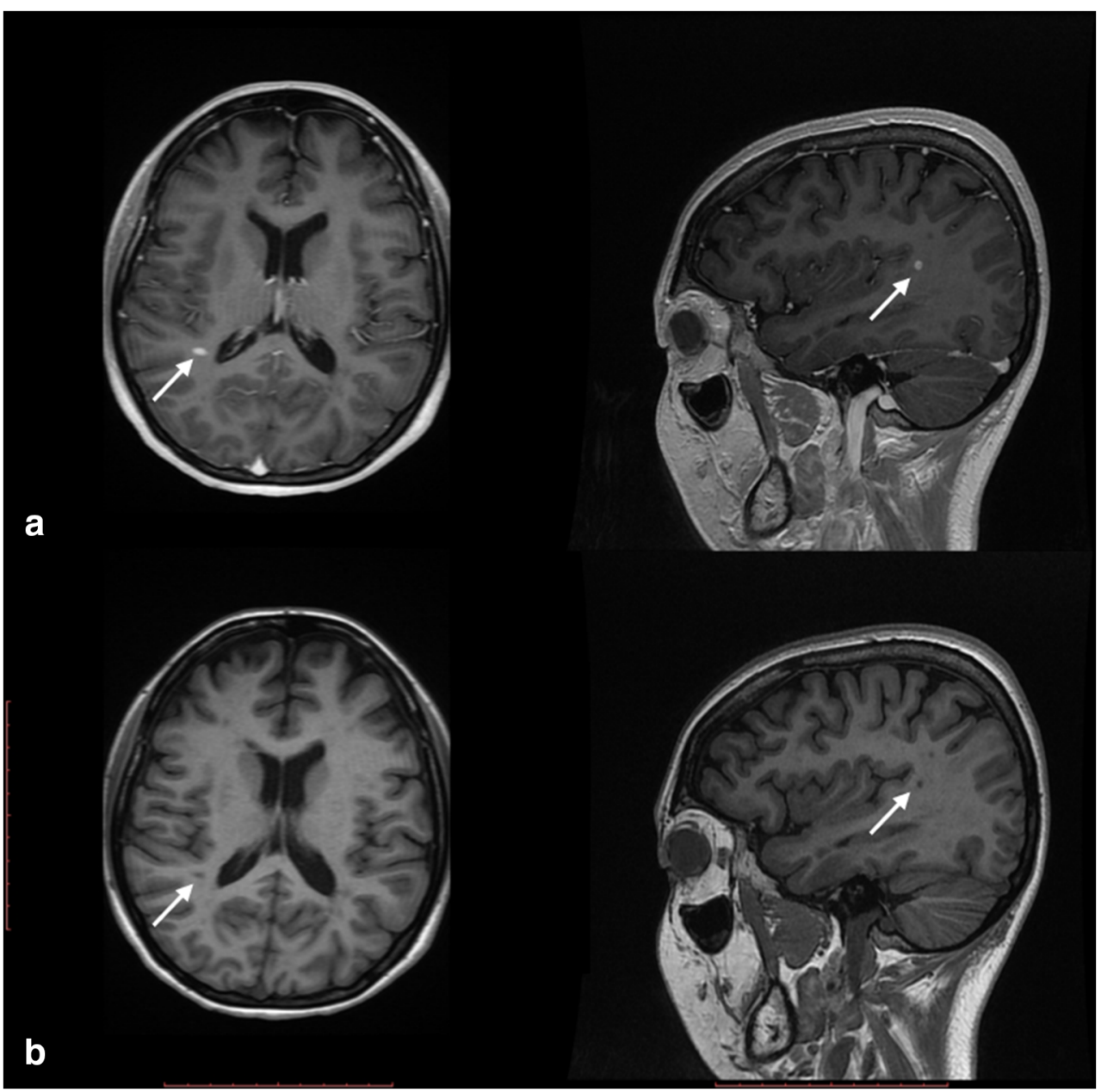


Fig. 5 a Cervical spine MRI T2 sequences (May 2011). Diffuse hyperintensities of poorly defined borders, in $\mathrm{C} 2-\mathrm{C} 3$ down to the inferior plate of $\mathrm{C} 5$ vertebral body (asterisk). b Cervical spine MRI T2 sequences (2012). Hypertense image in in medulla-spinal border down to the inferior plate of $\mathrm{C} 2$ (asterisk)

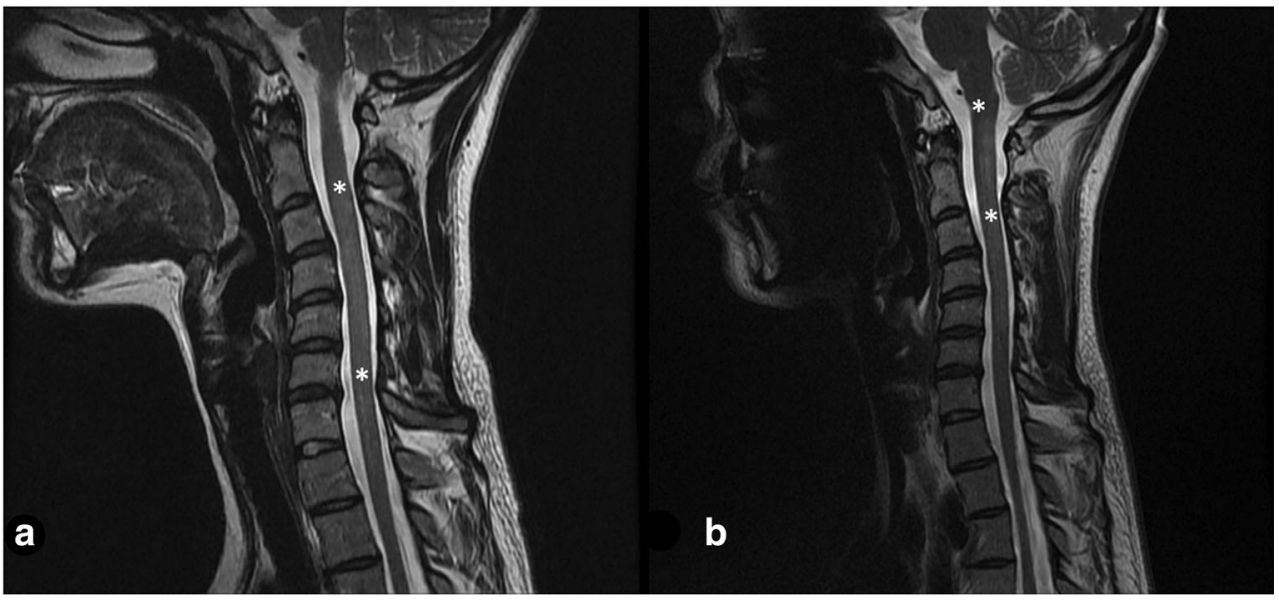

\section{Results and Discussion}

Linkage and Exome Analyses The maximized model for affected status segregation was that of recessive transmission with almost complete penetrance. Suggestive regions of linkage were defined by parametric and non-parametric LOD scores following standard criteria [24] on chromosomes: 1, $2,6,7,9,10,11,13,14$, and 15 (Table 3 ). Using the criterion of 1-LOD score, we defined the minimal critical regions (MCRs) containing the causal variants underpinning the linkage peak (Table 3). Using BioMart, an interface to retrieve data from Ensembl, we defined a total of 888 genes within MCRs (Table 3). We then performed a search for damaging variants from the 857,854 genomic variants that were identified in the seven individuals subjected to whole exome capture and sequencing. Among these, we retrieved novel and rare variants predicted to trigger functional consequences. We then determined whether these variants were homozygous in affected individuals and heterozygous in non-affected individuals (in agreement with the recessive model of transmission).

Functional homozygous variants were present in some interesting candidate genes (Table 4). The first candidate gene, diacylglycerol kinase iota ( $D G K I)$, is a member of the type IV diacylglycerol kinase subfamily. Diacylglycerol kinases regulate the intracellular concentration of diacylglycerol through its phosphorylation, producing phosphatidic acid [25]. Evaluation of a Drosophila homolog of DGK2, rdgA, showed retinal degeneration in homozygous $r d g A$ fruit flies [26]. Hozumi et al. showed that DGK1 in rat localizes to the outer plexiform layer, within which photoreceptor cells make contact with bipolar and horizontal cells [27]. The relationship of DGK1 with MS was described by Reich et al. [28]. Even though the specific role of this gene is unclear, Qiu et al. revealed that phosphorylation of Dgk1 by casein kinase II may play a crucial role in the production of phosphatidic acid in Saccharomyces cerevisiae [25].

Another candidate gene is TNF receptor superfamily member 10a (TNFRSF10A). This gene encodes a receptor for TNF family cytokines, which have a role in inflammation and immune regulation. This receptor is also known as DR4 and works as a receptor for TNF-related apoptosis-inducing ligand [29]. Variants in this gene are related to susceptibility of developing MS (rs4872077, OR of 1.34 95\% CI) [29, 30].

An additional candidate gene harboring variant was $\mathrm{G}$ protein subunit gamma transducin 1 (GNGT1). The protein encoded by the GNTG1 gene is specific to rod photoreceptors (defects in genes encoding proteins related to phototransduction can explain retinal defects) [31]. Recently, it was shown that $g n t g 1$ is expressed in the zebrafish retina and in other vertebrate species [32, 33].

C3 and PZP like, alpha-2-macroglobulin domain containing 8 (CPAMD8), is another gene involved in eye development and is associated with susceptibility to MS [34]. This gene encodes a member of the protease inhibitor I39 (alpha-2macroglobulin) family of proteins. Mutations in this gene cause an autosomal recessive developmental disorder of the eye, a form of anterior segment dysgenesis that includes ectopia lentis [35]. Alsaif et al. suggested that in these patients, congenital glaucoma appears to be part of the phenotype [36]. Mutations in CPAMD8 are described in Morganian cataract, an autosomal recessive congenital cataract that forms in red Holstein Friesian cattle [37].

Finally, another candidate gene is the TNF superfamily member 13b (TNFSF13B), also named B cell activating factor $(B A F F)$, which is associated with primary Sjögren syndrome susceptibility because of its capacity to induce antibody production [38]. Its expression is differentially regulated after transcorneal electrical stimulation. BARK, a gene upstream of BAFF, is involved in rhodopsin metabolism [39]. BAFF has been associated with autoimmunity risk; an Italian genome-wide association showed association with MS and systemic lupus erythematosus [40, 41]. B cells have an impact on MS, and Puthenparampil et al. suggested that BAFF might be absorbed by B cells that proliferate in the central nervous system of MS patients $[42,43]$. 
Table 3 Parametric and non-parametric estimated LOD scores in regions with suggestive and nominal genetic linkage with the list of genes contained in minimal critical regions defined by the 1-LOD score criterion

\begin{tabular}{|c|c|c|c|c|c|c|}
\hline $\mathrm{Chr}$ & Position & $\begin{array}{l}\text { 1-LOD } \\
\text { score } \\
\text { lower }\end{array}$ & $\begin{array}{l}\text { 1-LOD } \\
\text { score } \\
\text { upper }\end{array}$ & $\mathrm{LOD}_{\mathrm{MAX}}$ & $\begin{array}{l}\text { NPL } \\
\text { SPAIR }\end{array}$ & $\begin{array}{l}\text { NPL } \\
\text { SALL }\end{array}$ \\
\hline
\end{tabular}

$1 \quad 145112414 \quad 0.905867 \quad 1.470478 \quad 1.8063313 .4641023 .684484$ HIST2H3PS2, FAM72C, PPIAL4E, NBPF15, PPIAL4F, SRGAP2B, FAM72D, PPIAL4D, NBPF20

$2 \quad 130951584 \quad 0.9460751 .377104 \quad 1.805566 \quad 3.464102 \quad 3.684484$ ARHGEF4, AC009477.2, FAM168B, PLEKHB2, POTEE, WTH3DI, MZT2A, TUBA3D, CCDC74A, ANKRD3OBL, STAM2, FMNL2, PRPF40A, ARL6IP6

$\begin{array}{lllll}6 & 57467303 & 1.1247 & 1.4644 & 1.7774\end{array}$

RCC2P7, DST, AL512422.2, RNU6-626P, BEND6, OSTCP6, FTH1P15, KIAA1586, ZNF451, BAG2, RAB23, PRIM2, MIR548U, GUSBP4, POM121L14P, LINC00680, GAPDHP15, RBBP4P4

$\begin{array}{lllllll}7 & 128315882 & 1.230381 & 0.234805 & 1.805566 & 3.464102 & 3.684483\end{array}$

STEAP4, ZNF804B, TEX47, STEAP1, STEAP2, CFAP69, FAM237B, GTPBP10, CLDN12, CDK14, FZD1, MTERF1, AKAP9, CYP51A1, LRRD1, KRIT1, ANKIB1, GATAD1, ERVW-1, PEX1, RBM48, FAM133B, CDK6, SAMD9, SAMD9L, HEPACAM2, VPS50, CALCR, GNGT1, TFPI2, GNG11, BET1, COL1A2, CASD1, SGCE, PEG10, PPP1R9A, AC002429.2, PON1, PON3, PON2, ASB4, PDK4, DYNC111, SLC25A13, SEM1, DLX6, DLX5, SDHAF3, TAC1, ASNS, OCM2, LMTK2, BHLHA15, TECPR1, BRI3, BAIAP2L1, NPTX2, TMEM130, TRRAP, SMURF1, KPNA7, ARPC1A, ARPC1B, PDAP1, BUD31, PTCD1, ATP5J2-PTCD1, CPSF4, ATP5J2, ZNF789, ZNF394, ZKSCAN5, FAM200A, ZNF655, TMEM225B, ZSCAN25, CYP3A5, CYP3A7-CYP3A51P, CYP3A7, CYP3A4, CYP3A43, OR2AE1, TRIM4, GJC3, AZGP1, ZKSCAN1, ZSCAN21, ZNF3, COPS6, MCM7, AP4M1, TAF6, CNPY4, MBLAC1, LAMTOR4, C7orf43, GAL3ST4, GPC2, STAG3, GATS, PVRIG, SPDYE3, PILRB, PILRA, ZCWPW1, MEPCE, PPP1R35, C7orf61, TSC22D4, NYAP1, AGFG2, SAP25, LRCH4, FBXO24, PCOLCE, MOSPD3, TFR2, ACTL6B, GNB2, GIGYF1, POP7, EPO, ZAN, EPHB4, SLC12A9, TRIP6, SRRT, UFSP1, ACHE, MUC3A, MUC12, MUC17, TRIM56, SERPINE1, AP1S1, VGF, NAT16, MOGAT3, PLOD3, ZNHIT1, CLDN15, FIS1, IFT22, COL26A1, MYL10, CUX1, SH2B2, SPDYE6, PRKRIP1, ORAI2, ALKBH4, LRWD1, POLR2J, RASA4B, POLR2J3, SPDYE2, RASA4, UPK3BL1, POLR2J2, SPDYE2B, POLR2J2, FAM185A, FBXL13, LRRC17, NFE4, ARMC10, NAPEPLD, PMPCB, DNAJC2, PSMC2, SLC26A5, RELN, ORC5, LHFPL3, KMT2E, SRPK2, PUS7, RINT1, EFCAB10, ATXN7L1, CDHR3, SYPL1, NAMPT, CCDC71L, PIK3CG, PRKAR2B, HBP1, COG5, GPR22, DUS4L, BCAP29, SLC26A4, CBLL1, SLC26A3, DLD, LAMB1, LAMB4, NRCAM, PNPLA8, THAP5, DNAJB9, IMMP2L, LRRN3, DOCK4, ZNF277, IFRD1, LSMEM1, TMEM168, BMT2, GPR85, SMIM30, PPP1R3A, FOXP2, MDFIC, TFEC, TES, CAV2, CAV1, MET, CAPZA2, ST7, WNT2, ASZ1, CFTR, CTTNBP2, LSM8, ANKRD7, KCND2, TSPAN12, ING3, CPED1, WNT16, FAM3C, PTPRZ1, GCC1, KLF14, CCDC136, PARP12, TSPAN33, TBXAS1, LRGUK, MKRN1, BPGM, IRF5, SND1, LRRC4, CPA5, TMEM209, ZNF800, BRAF, STRA8, GRM8, C7orf77, CLEC2L, FAM71F2, FMC1, OPN1SW, CLEC5A, AC011005.1, CNOT4, PAX4, RAB19, SMO, FSCN3, ZC3HAV1L, ZC3HAV1, TNPO3, WDR91, DENND2A, ATP6V0A4, SVOPL, ADCK2, KCP, C7orf55-LUC7L2, GPR37, AKR1B15, POT1, AGBL3, OR9A4, TAS2R38, CEP41, TMEM213, AKR1B1, PLXNA4, TAS2R5, WEE2, C7orf49, MKLN1, AKR1B10, SMKR1, LUC7L2, ATP6V1F, FLNC, TMEM140, HIPK2, MEST, ARF5, SLC37A3, NDUFB2, SSU72P8, FAM71F1, CALD1, KDM7A, KLRG2, SLC35B4, SSBP1, CPA2, UBN2, PRSS37, ZC3HC1, AKR1D1, KIAA1147, NRF1, UBE2H, COPG2, TAS2R4, METTL2B, CPA1, HILPDA, KLHDC10, STRIP2, TRIM24, TAS2R3, KIAA1549, CALU, EXOC4, CHCHD3, MGAM, PODXL, TSGA13, TMEM178B, MRPS33, CREB3L2, AGK, CPA4, TTC26, IMPDH1, SSMEM1, LEP, NUP205, RBM28, AHCYL2, C7orf73, SLC13A4, FAM180A, LUZP6, MTPN, CHRM2, PTN, DGKI, PRRT4 
Table 3 (continued)

\begin{tabular}{lllllll}
\hline Chr Position & 1-LOD & 1-LOD & LOD $_{\text {MAX }}$ & NPL & NPL & Genes \\
& score & score & & SPAIR & SALL & \\
& lower & upper & & & & \\
& & & & & &
\end{tabular}

$10 \quad 46999151 \mathrm{~S} \quad 1.355892 \quad 1.507883 \quad 1.8052823 .4629683 .683198$ AKR1C2, AKR1C3, AKR1C4, UCN3, TUBAL3, NET1, PTPN20

$\begin{array}{lllll}11 & 104768909 & 1.2454 & 0.6977 & 1.8056\end{array}$

MUC6, MUC5AC, C11 orf88, BTG4, CASP1, DCUN1D5, MMP10, MMP7, POU2AF1, CASP5, FDX1, SLN, GUCY1A2, ARHGAP20, RDX, NPAT, ATM, ELMOD1, DYNC2H1, RAB39A, GRIA4, CUL5, ALKBH8, PDGFD, DDI1, LAYN, COLCA2, C11orf53, EXPH5, KBTBD3, MSANTD4, ZC3H12C, ANGPTL5, CWF19L2, AASDHPPT, CASP4, DDX10, SLC35F2, C11orf87, KDELC2, CARD17, MMP13, CARD18, MMP12, C11orf65, ACAT1, TMEM123, BIRC2, CARD16, YAP1, CEP126, MMP3, MMP8, C11 orf70, MMP1, MMP27, MMP20, PGR, TMEM133, BIRC3, TRPC6, ARHGAP42

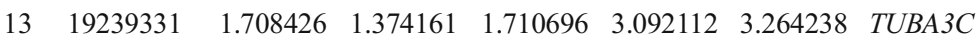

$1490730265 \quad 0.535392 \quad 1.433905 \quad 1.805566 \quad 3.464102 \quad 3.684484$ OR4N2, OR4K5, OR4K1, OR4K15, OR4K14, OR4K13, OR4L1, OR4K17, OR4N5, OR11G2, OR11H6, OR11H4, TTC5, CCNB1IP1, PARP2, TEP1, KLHL33, OSGEP, APEX1, TMEM55B, PNP, C140rf177, ATG2B, DIO3, SERPINA5, AL049839.2, DDX24, EVL, OTUB2, CDC42BPB, RPS6KA5, SYNE3, SLC24A4, ANKRD9, TECPR2, WDR20, SERPINA9, BCL11B, TMEM251, RIN3, TNFAIP2, NRDE2, WDR25, NDUFB1, CCDC85C, TTC7B, KCNK13, DICER1, EFCAB11, DLK1, BTBD7, DEGS2, SERPINA3, CCDC197, ASB2, VRK1, ZNF839, CCNK, TCL1A, TCL1B, MARK3, SETD3, CINP, DYNC1H1, SERPINA12, SERPINA11, IFI27L1, MOAP1, WARS, ITPK1, PAPOLA, SERPINA4, SLC25A47, YY1, PSMC1, EML1, AL161669.4, TRAF3, HHIPL1, AMN, GSKIP, CLMN, GSC, SLC25A29, RCOR1, TUNAR, HSP90AA1, CHGA, EIF5, C14orf132, UBR7, LGMN, CPSF2, C14orf159, AK7, GOLGA5, CALM1, BDKRB1, GLRX5, EXOC3L4, BDKRB2, MOK, CYP46A1, TDP1, PPP2R5C, BEGAIN, SERPINA1, IFI27L2, FAM181A, PRIMA1, UNC79, IFI27, PPP4R4, GON7, RTL1, SERPINA10, COX8C, CATSPERB, CCDC88C, SERPINA6, FBLN5, PPP4R3A, ATXN3, TRIP11, TC2N, GPR68, ASPG, RD3L, TMEM179, C14orf180, TDRD9, C14orf2, KIF26A

$151018277591.276506 \quad 1.804787 \quad 1.8055623 .4640853 .684465$ GOLGA8G, GOLGA6L7P, APBA2, ALDH1A3, LRRK1, OR4F4, OR4F6, TARSL2, CHSY1, SELENOS, PCSK6, OR4F15, TM2D3, SNRPA1

Table 4 List of novel and rare variants with functional effects in genes from the minimal critical regions cosegregating as homozygous in affected individuals (IU and/or MS) and heterozygous in unaffected individuals (in agreement with the recessive model of transmission). These variants are predicted to trigger functional consequences

\begin{tabular}{|c|c|c|c|c|c|c|c|c|c|c|}
\hline & Chromosome & Position & Gene & EMU-001 & EMU-002 & EMU-003 & EMU-004 & EMU-005 & $\begin{array}{l}\text { EMU- } \\
006\end{array}$ & EMU-007 \\
\hline Novel & $* 7$ & 137092781 & DGKI & $\mathrm{C}_{-} \mathrm{T}$ & C_C & C_C & C_C & C_C & $\mathrm{C}_{-} \mathrm{T}$ & $\mathrm{C}_{-} \mathrm{T}$ \\
\hline \multirow[t]{2}{*}{ Novels } & 7 & 93540348 & GNGT1 & G_T & G_G & G_G & G_G & G_G & G_T & G_G \\
\hline & $* 7$ & 137092781 & DGKI & $\mathrm{C}_{-} \mathrm{T}$ & C_C & C_C & C_C & C_C & $\mathrm{C}_{-} \mathrm{T}$ & $\mathrm{C}_{-} \mathrm{T}$ \\
\hline \multirow[t]{3}{*}{ Pathogenic } & 8 & 23059324 & TNFRSF10A & C_G & G_G & G_G & G_G & G_G & C_G & G_G \\
\hline & 8 & $23,060,256$ & TNFRSF10A & $\mathrm{C}_{-} \mathrm{T}$ & C_C & C_C & C_C & C_C & $\mathrm{C}_{-} \mathrm{T}$ & $\mathrm{C}_{-} \mathrm{C}$ \\
\hline & 19 & 17108135 & CPAMD 8 & $\mathrm{C}_{-} \mathrm{T}$ & $\mathrm{T}_{-} \mathrm{T}$ & $\mathrm{T}_{-} \mathrm{T}$ & $\mathrm{T}_{-} \mathrm{T}$ & $\mathrm{T}_{-} \mathrm{T}$ & $\mathrm{C}_{-} \mathrm{T}$ & $\mathrm{C}_{-} \mathrm{T}$ \\
\hline \multirow[t]{4}{*}{ Indels } & 7 & 103207412 & RELN & $\begin{array}{l}-- \\
\text { AAGGA- } \\
\text { AA }\end{array}$ & $\begin{array}{l}\text { AAGG } \\
\text { AAA- } \\
\text { AAGGA- } \\
\text { AA }\end{array}$ & $\begin{array}{l}\text { AAGG } \\
\text { AAA } \\
\text { AAGGA- } \\
\text { AA }\end{array}$ & $\begin{array}{l}\text { AAGG } \\
\text { AAA- } \\
\text { AAGGA- } \\
\text { AA }\end{array}$ & $\begin{array}{l}\text { AAGG } \\
\text { AAA- } \\
\text { AAGGA- } \\
\text { AA }\end{array}$ & $? \_?$ & $\begin{array}{l}{ }^{-} \\
\text {AAGGA- } \\
\text { AA }\end{array}$ \\
\hline & 7 & 103234986 & & $-{ }_{-} \mathrm{T}$ & $\mathrm{T}_{-} \mathrm{T}$ & $\mathrm{T}_{-} \mathrm{T}$ & $\mathrm{T}_{-} \mathrm{T}$ & $\mathrm{T}_{-} \mathrm{T}$ & $? ?$ & $-{ }_{-} \mathrm{T}$ \\
\hline & 7 & 103314360 & & -_CTC & CTC_CTC & CTC_CTC & CTC_CTC & CTC_CTC & ?_? & -_CTC \\
\hline & 13 & 108959081 & TNFSF13B & $-\_\mathrm{T}$ & $\mathrm{T}_{-} \mathrm{T}$ & $\mathrm{T}_{-} \mathrm{T}$ & $\mathrm{T}_{-} \mathrm{T}$ & $\mathrm{T}_{-} \mathrm{T}$ & ?_? & $\mathrm{T}_{-} \mathrm{T}$ \\
\hline
\end{tabular}


In conclusion, we have defined potential homozygous functional mutations that cosegregate in regions of either suggestive or nominal linkage with an autoimmune phenotype of IU and MS. Some of these variants are in candidate genes associated with ontogenetic processes of brain and eye differentiation. These variants, because of their pattern of expression, mostly in ocular and neurological tissues, warrant evaluation as causative alleles of these conditions in other families and in sporadic cases of IU and/or MS.

Acknowledgements We thank Jeremy Allen, $\mathrm{PhD}$, from Edanz Group (www.edanzediting.com/ac) for editing a draft of this manuscript and Humberto Madriñan Navia, MD, for the reading of the MRI.

\section{Compliance with Ethical Standards}

Conflict of Interest The authors declare that they have no conflict of interest.

Informed Consent Informed consent was obtained from all individual participants included in the study.

Statement on Human Ethics The procedures performed in our study involving human participants were in accordance with the ethical standards of the institutional research committee (Comité de ética en Investigación de la Escuela Superior de Oftalmología - Instituto Barraquer de América 009 26-11-2018) and with the 1964 Helsinki declaration and its later amendments. Informed consent was provided by all participants and parents provided informed consent for their daughter (younger than 16 years of age).

Open Access This article is distributed under the terms of the Creative Commons Attribution 4.0 International License (http:// creativecommons.org/licenses/by/4.0/), which permits unrestricted use, distribution, and reproduction in any medium, provided you give appropriate credit to the original author(s) and the source, provide a link to the Creative Commons license, and indicate if changes were made.

\section{References}

1. Jabs DA, Nussenblatt RB, Rosenbaum JT, Standardization of Uveitis Nomenclature (SUN) Working Group (2005) Standardization of uveitis nomenclature for reporting clinical data. Results of the First International Workshop. Am J Ophthalmol 140: 509-516

2. Acharya NR, Tham VM, Esterberg E, Borkar DS, Parker JV, Vinoya AC, Uchida A (2013) Incidence and prevalence of uveitis: results from the Pacific Ocular Inflammation Study. JAMA Ophthalmol 131:1405-1412.

3. Miserocchi E, Fogliato G, Modorati G, Bandello F (2013) Review on the worldwide epidemiology of uveitis. Eur J Ophthalmol 23: 705-717

4. Krishna U, Ajanaku D, Denniston AK, Gkika T (2017) Uveitis: a sight-threatening disease which can impact all systems. Postgrad Med J 93:766-773

5. Levy RA, de Andrade FA, Foeldvari I (2011) Cutting-edge issues in autoimmune uveitis. Clin Rev Allergy Immunol 41:214-223

6. Babu BM, Rathinam SR (2010) Intermediate uveitis. Indian J Ophthalmol 58:21-27.
7. Gueudry J, Muraine M (2018) Anterior uveitis. J Fr Ophtalmol 41: e11-e21

8. Hoy SM (2017) Adalimumab: a review in non-infectious non-anterior uveitis. BioDrugs 31:135-142

9. Uy HS, Yu-Keh E, Chan PS (2016) Posterior uveitis. Dev Ophthalmol 55:163-166

10. Hou S, Kijlstra A, Yang P (2015) Molecular genetic advances in uveitis. Prog Mol Biol Transl Sci 134:283-298

11. Pichi F, Carrai P, Srivastava SK, Lowder CY, Nucci P, Neri P (2016) Genetic of uveitis. Int Ophthalmol 36:419-433

12. Yang MM, Lai TY, Luk FO, Pang CP (2014) The roles of genetic factors in uveitis and their clinical significance. Retina 34(1):1-11

13. Martin TM, Kurz DE, Rosenbaum JT (2003) Genetics of uveitis. Ophthalmol Clin N Am 16:555-565

14. Bonfioli AA, Damico FM, Curi AL, Orefice F (2005) Intermediate uveitis. Semin Ophthalmol 20:147-154

15. Le Scanff J, Sève P, Renoux C, Broussolle C, Confavreux C, Vukusic S (2008) Uveitis associated with multiple sclerosis (2008) uveitis associated with multiple sclerosis. Mult Scler 14: 415-417

16. Olsen TG, Frederiksen J (2017) The association between multiple sclerosis and uveitis. Surv Ophthalmol 62:89-95

17. Przeździecka-Dołyk J, Węgrzyn A, Turno-Kręcicka A, MisiukHojło M (2016) Immunopathogenic background of pars Planitis. Arch Immunol Ther Exp 64:127-137

18. Atan D, Heissigerova J, Kuffová L, Hogan A, Kilmartin DJ, Forrester JV, Bidwell JL, Dick AD et al (2013) Tumor necrosis factor polymorphisms associated with tumor necrosis factor production influence the risk of idiopathic intermediate uveitis. Mol Vis 19:184-195

19. Li H (2013) Aligning sequence reads, clone sequences and assembly contigs with BWA-MEM. eprint arXiv:1303.3997.

20. McKenna A, Hanna M, Banks E, Sivachenko A, Cibulskis K, Kernytsky A, Garimella K, Altshuler D et al (2010) The Genome Analysis Toolkit: a MapReduce framework for analyzing nextgeneration DNA sequencing data. Genome Res 20:1297-1303

21. Van der Auwera GA et al (2013) Van der Auwera GA, Carneiro MO, Hartl C, Poplin R, Del Angel G, Levy-Moonshine A, Jordan T, Shakir K, Roazen D, Thibault J, Banks E, Garimella KV, Altshuler D, Gabriel S, DePristo MA. From FastQ data to high confidence variant calls: the Genome Analysis Toolkit best practices pipeline. Curr Protoc Bioinformatics 11:11 10 1-11 1033.

22. DePristo MA, Banks E, Poplin R, Garimella KV, Maguire JR, Hartl C, Philippakis AA, del Angel G et al (2011) A framework for variation discovery and genotyping using next-generation DNA sequencing data. Nat Genet 43:491-498

23. Raza MH, Gertz EM, Mundorff J, Lukong J, Kuster J, Schäffer AA, Drayna D (2013) Linkage analysis of a large African family segregating stuttering suggests polygenic inheritance. Hum Genet 132: 385-396

24. Lander E, Kruglyak L (1995) Genetic dissection of complex traits: guidelines for interpreting and reporting linkage results. Nat Genet 11:241-247

25. Qiu Y, Hassaninasab A, Han GS, Carman GM (2016) Phosphorylation of Dgk1 diacylglycerol kinase by casein kinase II regulates phosphatidic acid production in Saccharomyces cerevisiae. J Biol Chem 291:26455-26467

26. Bowne SJ, Sullivan LS, Ding L, Traer E, Prescott SM, Birch DG, Kennan A, Humphries P et al (2000) Evaluation of human diacylglycerol kinase (iota), DGKI, a homolog of Drosophila rdgA, in inherited retinopathy mapping to 7q. Mol Vis 6:6-9

27. Hozumi Y, Matsui H, Sakane F, Watanabe M, Goto K (2013) Distinct expression and localization of diacylglycerol kinase isozymes in rat retina. J Histochem Cytochem 61:462-476

28. Reich D, Patterson N, De Jager PL, McDonald GJ, Waliszewska A, Tandon A, Lincoln RR, DeLoa C et al (2005) A whole-genome 
admixture scan finds a candidate locus for multiple sclerosis susceptibility. Nat Genet 37:1113-1118

29. Cannella B, Gaupp S, Omari KM, Raine CS (2007) Multiple sclerosis: death receptor expression and oligodendrocyte apoptosis in established lesions. J Neuroimmunol 188:128-137

30. López-Gómez C, Fernández O, García-León JA, Pinto-Medel MJ, Oliver-Martos B, Ortega-Pinazo J, Suardíaz M, García-Trujillo L et al (2011) TRAIL/TRAIL receptor system and susceptibility to multiple sclerosis. PLoS One 6:e21766

31. Scherer SW, Feinstein DS, Oliveira L, Tsui LC, Pittler SJ (1996) Gene structure and chromosome localization to $7 \mathrm{q} 21.3$ of the human rod photoreceptor transducin gamma-subunit gene (GNGT1). Genomics 35:241-243

32. Lagman D, Callado-Pérez A, Franzén IE, Larhammar D, Abalo XM (2015) Transducin duplicates in the zebrafish retina and pineal complex: differential specialisation after the teleost tetraploidisation. PLoS One 10:e121330

33. Chen H, Leung T, Giger KE, Stauffer AM, Humbert JE, Sinha S, Horstick EJ, Hansen CA et al (2007) Expression of the G protein gammaT1 subunit during zebrafish development. Gene Expr Patterns 7:574-583

34. Baranzini SE, Wang J, Gibson RA, Galwey N, Naegelin Y, Barkhof F, Radue EW, Lindberg RL et al (2009) Genome-wide association analysis of susceptibility and clinical phenotype in multiple sclerosis. Hum Mol Genet 18:767-778

35. Cheong SS, Hentschel L, Davidson AE, Gerrelli D, Davie R, Rizzo R, Pontikos N, Plagnol V et al (2016) Mutations in CPAMD8 cause a unique form of autosomal-recessive anterior segment dysgenesis. Am J Hum Genet 99:1338-1352

36. Alsaif HS et al (2018) Congenital glaucoma and CYP1B1: an old story revisited. Hum Genet p:1-7
37. Hollmann AK, Dammann I, Wemheuer WM, Wemheuer WE, Chilla A, Tipold A, Schulz-Schaeffer WJ, Beck J et al (2017) Morgagnian cataract resulting from a naturally occurring nonsense mutation elucidates a role of CPAMD8 in mammalian lens development. PLoS One 12:e180665

38. Deng F, Chen J, Zheng J, Chen Y, Huang R, Yin J, Gao X, Lin Q et al (2016) Association of BAFF and IL-17A with subphenotypes of primary Sjogren's syndrome. Int J Rheum Dis 19:715-720

39. Deng F, Chen J, Zheng J, Chen Y, Huang R, Yin J, Gao X, Lin Q et al (2004) Rhodopsin kinase activity modulates the amplitude of the visual response in Drosophila. Proc Natl Acad Sci U S A 101: 11874-11879

40. Steri M, Orrù V, Idda ML, Pitzalis M, Pala M, Zara I, Sidore C, Faà $\mathrm{V}$ et al (2017) Overexpression of the cytokine BAFF and autoimmunity risk. N Engl J Med 376:1615-1626

41. Pandit L, Ban M, Beecham AH, McCauley JL, Sawcer S, D'Cunha A, Malli C, Malik O (2016) European multiple sclerosis risk variants in the south Asian population. Mult Scler 22:1536-1540

42. Puthenparampil M, Miante S, Federle L, Zanetta C, Toffanin E, Ruggero S, Rinaldi F, Gallo P (2016) BAFF is decreased in the cerebrospinal fluid of multiple sclerosis at clinical onset. J Neuroimmunol 297:63-67

43. Puthenparampil M, Federle L, Miante S, Zito A, Toffanin E, Ruggero S, Ermani M, Pravato S et al (2017) BAFF index and CXCL13 levels in the cerebrospinal fluid associate respectively with intrathecal IgG synthesis and cortical atrophy in multiple sclerosis at clinical onset. J Neuroinflammation 14:11

Publisher's Note Springer Nature remains neutral with regard to jurisdictional claims in published maps and institutional affiliations. 